\title{
Prevalência e Fatores Correlatos de Infecção pelo HIV e Sífilis em Prostitutas Atendidas em Centro de Referência DST/AIDS
}

\author{
Prevalence and Correlates of HIV Infection and Syphilis in \\ Prostitutes Attending a STD/AIDS Reference Center
}

Isabel Cristina Pinheiro Pires, Angélica Espinosa Barbosa Miranda

\section{RESUM0}

Um estudo retrospectivo foi conduzido com análise dos prontuários médicos de prostitutas atendidas no Centro de Referência para DST/AIDS em Vitória (ES) no periodo de janeiro de 1993 a dezembro de 1996. Durante este periodo, 180 mulheres receberam atendimento médico e psicológico nesta clínica. A média de idade foi de 25,9 anos (DP =6,8). De um total de 180 mulheres, 140 concordaram em serem testadas para HIV, das quais 12 (8,6\%) apresentaram resultado positivo. De 157 mulheres que concordaram em serem testadas para sífilis, 13 (8,3\%) apresentaramo VDRL positivo. Quanto ao nível de educação, 6 mulheres $(3,3 \%)$ eram analfabetas, 114 (63,3\%) completaram o primeiro grau, 37 (20,6\%) estudaram até o segundo grau, 7 (3,9\%) estavam na universidade e $16(8,9 \%)$ não quiseram informar. Quanto ao estado civil, 141 (78,3\%) eram solteiras, 17 (9,4\%) casadas, 10 (5,5\%) divorciadas e 4 (2,2\%) viúvas. Quanto à freqüência do uso de condom, $56(31,3 \%)$ relataram que sempre usavam, 93 (52,0\%) às vezes e 30 (16,8\%) nunca usavam. Doenças sexualmente transmissiveis (DST) prévias foram relatadas por 89 mulheres $(49,4 \%)$ e $46(25,6 \%)$ apresentavam alguma DST na ocasião da consulta. Nove mulheres $(5,0 \%)$ relataram uso de drogas injetáveis. Houve diferença estatisticamente significante entre o grupo com sorologia para HIV negativo e o positivo quando se comparou o uso de drogas injetáveis ( $p=0,031$ ) e a infecção por sífilis ( $p=0,014)$. O presente estudo mostrou que as taxas de prevalência da infecção pelo HIV em trabalhadoras do sexo são mais altas que as encontradas na população em geral. Isto aponta para a necessidade de reforçar a assistência médica e campanhas educativas, especialmente direcionadas para esta população de mulheres, abordando a importância do uso regular do preservativo e dos riscos associados ao uso de drogas injetáveis.

PALAVRAS-CHAVE: Prostituição. AIDS. Sífilis. Doenças sexualmente transmissíveis. Drogadição. Atendimento primário.

Centro de Referência para DST/AIDS - Prefeitura Municipal 
Introdução

O número de mulheres contaminadas pelo HIV tem aumentado em todo mundo. Muitos fatores contribuem para a vulnerabilidade diante do HIV, desde fatores biológicos e culturais até fatores sociais e econômicos. Neste panorama, as trabalhadoras do sexo apresentam o agravante de sua condição "marginal" na sociedade, o que faz com que tenham dificuldade no acesso a serviços de saúde e programas de prevenção. Elas são consideradas reservatórios de infecção e não como recipientes em potencial, com risco de adoecer ${ }^{9}$.

As políticas de saúde para esta população envolvem quase sempre diversas tentativas de controle médico e estatal, como se a testagem compulsória para o HIV ou a determinação de espaços específicos para exercerem sua atividade garantissem o controle do vírus ${ }^{6}$.

As altas taxas de infecção por HIV e outras DST apontadas pelas pesquisas de soroprevalência realizadas entre trabalhadoras do sexo em vários países indicam que os programas de prevenção e assistência para esta população são inexistentes ou ineficientes. Uma das principais dificuldades para a implementação dessas ações é a resistência usual destas mulheres à abordagem e ao acompanhamento pelos serviços de saúde. Assim, para se alcançar êxito nestes programas é importante conhecer o perfil da população alvo, seu modo de vida e suas práticas de risco, direcionando-se as ações ao encontro de suas características e necessidades próprias ${ }^{10}$.

Na Grande Vitória há um crescente mercado consumidor para o produto oferecido pelas trabalhadoras do sexo. Isto é explicado em parte pela geografia local, uma região cortada por duas rodovias federais com intenso tráfego de caminhões e com um estratégico litoral que abriga cinco portos, o que inunda suas ruas com marinheiros de toda parte do mundo. As profissionais do sexo oferecem, então, seus serviços nas ruas e bares, em inúmeros Nights Clubs, boates e saunas, além de anúncios nos jornais locais.

O objetivo deste estudo foi conhecer de forma sistematizada o perfil e determinar a soroprevalência da infecção pelo HIV e sífilis das trabalhadoras do sexo atendidas no Centro de Referência, para melhor atuar na abordagem desta população e implementar programas de prevenção e assistência.

\section{Material e Métodos}

Foi realizado um estudo retrospectivo avaliando os prontuários médicos de todas trabalhadoras do sexo atendidas no Centro de
Referência em DST/AIDS da Prefeitura Municipal de Vitória durante o período de janeiro de 1993 a dezembro de 1996. As informações colhidas no questionário foram: dados sociodemográficos, comportamento sexual das pacientes, uso de preservativos, uso de drogas injetáveis, transfusão sangüinea e história de DST.

O Centro de Referência em DST/AIDS, neste período, atendeu 180 prostitutas que procuraram o serviço espontaneamente ou mediante encaminhamento médico. Estas pacientes foram submetidas previamente aos testes sorológicos a uma consulta de orientação sobre a infecção pelo HIV e os exames a serem realizados. Nesta consulta também foram colhidos dados demográficos e informações sobre comportamentos de risco através de um questionário estruturado padronizado no serviço. Somente após este contato inicial e a concordância da paciente foram realizados os testes anti-HIV e VDRL.

O diagnóstico foi estabelecido por meio da realização de dois testes ELISA e confirmado pela reação de imunofluorescência indireta para HIV e VDRL para sífilis. Todas as pacientes foram convidadas a fazer o controle psicológico e ginecológico no CR DST/AIDS independentemente da realização da sorologia para HIV.

\section{Resultados}

No período estudado, 180 trabalhadoras do sexo receberam atendimento ginecológico e psicológico neste serviço. A idade média foi de 25,9 anos (desvio padrão: 6,8 anos). Das 180, 140 concordaram em serem testadas para o HIV (indice de aceitação de 77,8\%) e destas, 12 (8,6\%) apresentaram resultado positivo. De 157 que concordaram em serem testadas para a sífilis (indice de aceitação de 87,2\%), 144 (91,7\%) tiveram resultado negativo, ao passo que $13(8,3 \%)$ tiveram resultado positivo.

Quanto à escolaridade, 6 (3,3\%) eram analfabetas, $144(63,3 \%)$ tinham até o $1^{\circ}$ grau, 37 $(20,6 \%)$ tinham até o $2^{\circ}$ grau, $7(3,9 \%)$ estavam na universidade e $16(8,9 \%)$ não relataram a escolaridade. Cento e quarenta e uma pacientes $(78,3 \%)$ eram solteiras, $17(9,4 \%)$ casadas, 10 $(5,6 \%)$ separadas e $4(2,2 \%)$ viúvas.

A freqüência do uso do preservativo foi: 56 $(31,3 \%)$ o usavam sempre; 93 (52\%), às vezes e 30 $(16,8 \%)$ nunca usaram preservativo. Em relação ao número de parceiros sexuais, $79 \%$ das mulheres relataram mais de 10 parceiros por semana. Oitenta e nove mulheres $(49,4 \%)$ relataram história prévia de DST e $46(25,6 \%)$ apresentavam alguma DST na ocasião da consulta. Nove mulheres (5\%) 
relataram uso de drogas injetáveis. De sessenta e quatro mulheres, correspondendo a $35,6 \%$ dos casos, das quais foi colhida citologia cervicovaginal encontrou-se classe III de Papanicolaou em 6 $(9,4 \%)$, sendo que em 3 destes casos as alterações foram associadas à infecção pelo HPV.

Houve uma diferença significativa entre o grupo positivo e o negativo para o HIV em relação ao uso de drogas injetáveis $(\mathrm{p}=0,031)$ e infecção pela sífilis $(\mathrm{p}=0,014)$.

\section{Discussão}

Em razão do papel predominante da transmissão heterossexual na epidemia do HIV em muitos países, não é surpreendente que as profissionais do sexo e seus clientes desempenhem um papel importante nesta epidemia ${ }^{10}$. Tem-se observado uma grande variabilidade mundial da soroprevalência de HIV nesta população. Esta variabilidade guarda relação com a idade, grau de escolaridade, estado civil, número de clientes, local de trabalho, tempo de dedicação ao ofício, uso de preservativos, antecedentes de DST e abuso de drogas $^{4,14}$.

Em trabalhos realizados em países da África foram observadas prevalências que variaram de 10 a $30 \%{ }^{5}$. Em amostras da América Latina e do Caribe foram encontrados desde índices de 1\% na América do Sul até $40 \%$ no Haiti e Martinica ${ }^{10}$. Zapiola et al. encontraram uma prevalência de $6,3 \%$ em Buenos Aires ${ }^{16}$. Em Georgetown, Guiana, a prevalência foi de $25 \%{ }^{2}$. Em estudos realizados na Europa, a prevalência variou entre 1 a $6 \%^{11,13,15}$.

No Brasil não há dados que mostrem a verdadeira situação da infecção pelo HIV nessa população. Há apenas dados isolados de serviços que atendem pessoas com DST. Em trabalho realizado pela Faculdade de Medicina de Sorocaba foi encontrada uma prevalência de $12,9 \%$ de infecção pelo HIV e de 3,3\% de sífilis ${ }^{1}$. Cortes et al. encontraram uma prevalência de $9 \%$ em prostitutas de cidades de Minas Gerais ${ }^{3}$.

Em estudos realizados em outras populações de nossa região foram encontrados índices de 0,8\% de infecção pelo HIV e de $2,9 \%$ de sífilis em gestantes $^{8} ; 6$ e 14\% de infecção pelo HIV e sífilis, respectivamente, em mulheres que procuraram atendimento em clínica de $\mathrm{DST}^{7}$.

Muitas são as dificuldades enfrentadas pelos serviços de saúde para abordar a população de trabalhadoras do sexo. As dificuldades são tanto de estruturação dos serviços para se adequarem à realidade e às necessidades da população alvo, bem como em relação à sua permanência no atendimento pela insegurança inerente à condição de trabalhadora do sexo (medo da polícia, medo de ser rotulada de doente e ser impedida de trabalhar, estigma social, etc) ${ }^{6}$.

Uma revisão das políticas sociais e econômicas que dizem respeito à industria do sexo e suas conseqüências para a Saúde Pública mostram o papel significante do controle das DST e do abuso de drogas injetáveis nesta população. Educação sobre práticas de sexo seguro e controle do uso de drogas injetáveis deveriam ser incluídos em programas de prevenção. Medidas de prevenção e tratamentos sindrômico e epidemiológico devem ser oferecidos e deve ser garantido o acesso aos serviços de saúde em geral, inclusive a seus filhos, fomentando sua permanência nos serviços, com melhor resultado não só para o controle das DST, mas da saúde em geral ${ }^{12}$.

\section{SUMMARY}

A retrospective study examining medical records of female prostitutes attending the STD/AIDS Reference Center in Vitória, Brazil from January/93 to December/96 was conducted. During this period, 180 women received medical and psychological care in this clinic. Mean age was 25.9 year (SD=6.8). Out of 180, 140 agreed to be tested for HIV, of whom $12(8.6 \%)$ had a positive result. Among 157 women who agreed to be tested for syphilis, 144 (91.7\%) had a negative result, while 13 (8.3\%) had a positive one. According to the educational degree, 6 (3.3\%) women were illiterate, $114(63.3 \%)$ attended elementary school, $37(20.6 \%)$ attended secondary school, 7 (3.9\%) went to college and 16 gave no information. One hundred and forty-one patients (78.3\%) were single, $17(9.4 \%)$ married, $10(5,5 \%)$ divorced and $4(2.2 \%)$ widows. The frequency of condom use was: always, 56 $(31.3 \%)$, sometimes, $93(52.0 \%)$, and $30(16.8 \%)$ never used condoms. Other STDs were reported by 89 (49.4\%) women and $9(5.0 \%)$ reported intravenous (IV) drug use. There was a significant difference between the HIV positive and the negative group only regarding IV drug abuse $(p=0.031)$ and syphilis infection $(p=0.014)$. The present study showed prevalence rates of HIV infection among prostitutes in Vitória much higher than those found in the general population. There is a pressing need to improve medical assistance and educational campaigns especially designed to reach this population of women, and focusing the importance of regular condom use and the risks associated with IV drug abuse.

KEYS WORDS: Prostitution. AIDS. Syphilis. STD. Drug addiction. Primary care. 


\section{Referências bibliográficas}

1. Cançado, RR, Faria M, Ferreira RMV, Dias Jr JA, Reis E, Epiphanio MG. et al. Estudo da prevalência de doenças sexualmente transmissiveis em grupos de alto risco. Anais do VI Congresso Brasileiro de DST/ AIDS; 1996, Porto Alegre.

2. Carter KH, Harry BP, Jeune M, Nicholson D. HIV risk perception, risk behavior, and seroprevalence among female commercial sex workers in Georgetown, Guyana. Rev Panam Salud Publ 1997; 1: 451-9.

3. Cortes E, Detels R, Aboulafia D, Li XL, Moudgil T, Alam M, Bonecker C, Gonzaga A, Oyafuso L, Tondo M. HIV-1, HIV-2, and HTLV-I infection in high-risk groups in Brazil. N Engl J Med 1989; 320: 953-8.

4. Jackson L, Highcrest A, Coates RA. Varied potential risks of HIV infection among prostitutes. Soc Sci Med 1992; 35:281-6.

5. Larson A. Social context of HIV transmission in Africa: historical and cultural basis of East and Central African sexual relations. Rev Infect Dis 1989; 11: 716-31.

6. Mann J, Tarantola DJM, Netter TW. AIDS no mundo. Rio de Janeiro: Relume Dumará; 1993.

7. Miranda AE, Friço AR. Prevalência e fatores de risco em mulheres HIV+/AIDS atendidas em Centro de Referência em DST/AIDS. Rev Bras Ginecol Obstet 1997;19: $275-8$.
8. Miranda AE, Tonini RC, Alves MC, Friço AR, Ferreira $\mathrm{BM}$, Foletto SR, et al. Seroprevalence and risk factors of HIV infection in pregnant women in Vitória, Brazil. International Congress of Sexually Transmitted Diseases, 1997; Seville, Spain. p 419.

9. O’Leary S, Cheney B, Dossiê panos. Tripla Ameaça: AIDS e mulheres, tradução Ana Dourado. $1^{\mathrm{a}}$ ed. Rio de Janeiro: ABIA; 1993. p. 91-113.

10.Padian N. Prostitute women and AIDS: epidemiology. AIDS 1988; 2: 413-9.

11.Papaevangelou G, Roumeliotou-Karayannis A, Kallinikos G, Papoutsakis G. LAV/HTLV-III in female prostitutes. (Letter). Lancet 1985; 2:1018.

12.Piot P, Laga M. Prostitutes: a high risk group for HIV infection? Soz Praventiv Med 1988; 33:336-9.

13.Schultz S, Milberg JA, Kristal AR, Stoneburner RL. Female to male transmission of HTLV-III. (Letter). JAMA 1986; 255: 1703-4.

14.Simonsen JN, Plummer FA, Ngugi EN. HIV infection among lower socioeconomic strata prostitutes in Nairobi. AIDS 1990; 4:139-44.

15.Smith GL, Smith KF. Lack of HIV infection and condom use in licensed prostitutes. (Letter). Lancet 1986; 2: 1392.

16.Zapiola I, Salomone S, Alvarez A, Scolastico MC, Koessel RA, Lemus J, et al. HIV-1, HIV-2, HTLV-I/II and STD among female prostitutes in Buenos Aires, Argentina. Eur J Epidemiol 1996; 12: 27-31. 